\title{
microRNA-195 inhibits cell proliferation in bladder cancer via inhibition of cell division control protein 42 homolog/signal transducer and activator of transcription-3 signaling
}

\author{
CHENG ZHAO $^{1}$, LIN QI $^{1}$, MINFENG CHEN ${ }^{1}$, LONGFEI LIU ${ }^{1}$, \\ WEIQIAN YAN ${ }^{2}$, SHIYU TONG ${ }^{1}$ and XIONGBING ZU ${ }^{1}$ \\ Departments of ${ }^{1}$ Urology and ${ }^{2}$ Neurology, Xiangya Hospital of Central South University, \\ Changsha, Hunan 410008, P.R. China
}

Received May 21, 2014; Accepted February 4, 2015

DOI: $10.3892 / \mathrm{etm} .2015 .2633$

\begin{abstract}
RNA (miR)-195 acts as a suppressor in multiple types of malignant tumors, including bladder cancer; however, the detailed function of miR-195 in bladder cancer remains largely unknown. The aim of the present study was to investigate the role of miR-195 in the regulation of bladder cancer cell proliferation and to determine whether cell division control protein 42 homolog (Cdc42)/signal transducer and activator of transcription-3 (STAT3) signaling acts as a downstream effector of miR-195 in bladder cancer cells. Reverse transcription-quantitative polymerase chain reaction was used to determine the expression levels of miR-195 in bladder cancer tissues and normal adjacent tissue. The results revealed that the expression of miR-195 was significantly downregulated in bladder cancer tissues compared with that in the normal adjacent tissues. A luciferase reporter assay was then conducted, which identified Cdc42 as a direct target of miR-195, and the expression of Cdc42 was significantly upregulated in bladder cancer tissues, as determined by western blotting. Furthermore, miR-195 negatively regulated the protein expression of $\mathrm{Cdc} 42$ in bladder cancer cells. An MTT assay was also conducted to determine the rate of cell proliferation. Upregulation of miR-195 or the inhibition of Cdc42 could inhibit bladder cancer cell proliferation, possibly through activation of STAT3 signaling. In addition, restoration of $\mathrm{Cdc} 42$ could reverse the inhibitory effect of miR-195 upregulation on bladder cancer cell proliferation. In conclusion, the results of the present study suggest that miR-195 plays an inhibitory role in the regula-
\end{abstract}

Correspondence to: Professor Lin Qi, Department of Urology, Xiangya Hospital of Central South University, 88 Xiangya Road, Changsha, Hunan 410008, P.R. China

E-mail: csulinqi@163.com

Key words: bladder cancer, microRNA-195, cell division control protein 42 homolog, proliferation tion of bladder cancer cell proliferation by directly targeting Cdc42/STAT3 signaling.

\section{Introduction}

As one of the most common malignant cancers, bladder cancer severely threatens the health of individuals worldwide. Downregulation of tumor suppressors has been demonstrated to largely contribute to the development and progression of bladder cancer $(1,2)$. The development of effective gene targets for the treatment of bladder cancer is therefore urgently required.

microRNAs (miRNAs) are small (18-25 nucleotides in length), endogenous, non-coding RNAs that can lead to the silencing of gene expression by directly binding to specific seed sequences in the 3'-untranslated region (UTR) of their target RNAs (3). A number of miRNAs have been shown to act as key regulators in bladder cancer, including miRNA (miR)-145, miR-143, miR-183, miR-96 and miR-195 (4,5). Among these miRNAs, miR-195 has been shown to act as a suppressor in various cancers $(4,6)$; for example, miR-195 suppressed the proliferation, migration and invasion of non-small cell lung cancer by targeting MYB (7). miR-195 has also been suggested to be useful as a potential diagnostic and therapeutic target for breast cancer $(8,9)$, and has been shown to inhibit bladder cancer cell proliferation by directly targeting CDK4 and GLUT3 $(10,11)$. As one miRNA has multiple targets, and one mRNA can be targeted by numerous miRNAs (12), the underlying molecular mechanisms of miR-195 in bladder cancer, and the existence of other targets of miR-195, remain unclear.

Cell division control protein 42 homolog (Cdc42), a small GTPase of the Rho-subfamily, participates in the regulation of several signaling pathways that control diverse cellular biological processes, including cell migration, endocytosis and cell cycle progression (13). The association between Cdc42 and miRNAs in bladder cancer remains largely unclear. The aim of the present study, therefore, was to investigate the role of miR-195 in the regulation of bladder cancer cell prolif- 
eration and to determine whether $\mathrm{Cdc} 42 /$ signal transducer and activator of transcription-3 (STAT3) signaling acts as a downstream effector of miR-195 in bladder cancer cells.

\section{Materials and methods}

Tissue specimen collection. The present study was approved by the Ethics Committee of Central South University (Changsha, China). Informed consent was obtained from each of the patients in the study. Eighteen bladder cancer tissues, in addition to their matched adjacent normal tissues, were collected in the Department of Urinary Surgery, Xiangya Hospital of Central South University. Tissue samples were immediately frozen in liquid nitrogen following surgical removal.

Cell culture. Human bladder cancer T24 cells were obtained from the Cell Bank of Central South University, and cultured in Dulbecco's modified Eagle's medium (Gibco Life Technologies, Carlsbad, CA, USA) with $10 \%$ fetal bovine serum (Gibco Life Technologies) at $37^{\circ} \mathrm{C}$ in a humidified incubator containing $5 \% \mathrm{CO}_{2}$.

Reverse transcription-quantitative polymerase chain reaction $(R T-q P C R)$ assay. Total RNA was extracted using TRIzol ${ }^{\circledR}$ reagent (Invitrogen Life Technologies, Carlsbad, CA, USA), according to the manufacturer's instructions. For the analysis of miRNA expression, TaqMan ${ }^{\circledR}$ MicroRNA Reverse Transcription kit (Invitrogen Life Technologies) was used to convert RNA into cDNA, in accordance with the manufacturer's instructions. qPCR was then performed using All-in-One ${ }^{\mathrm{TM}}$ miRNA qPCR Detection kit (GeneCopoeia, Rockville, MD, USA) on an ABI 7500 thermocycler (Applied Biosystems, Foster City, CA, USA). The PCR cycling conditions were set as follows: $95^{\circ} \mathrm{C}$ for $3 \mathrm{~min}$, followed by 40 cycles of $95^{\circ} \mathrm{C}$ for $15 \mathrm{sec}$ and $60^{\circ} \mathrm{C}$ for $15 \mathrm{sec}$. U6 was used as a reference gene. The relative expression was analyzed by the $2^{-\Delta \Delta \mathrm{Ct}}$ method.

Western blot analysis. Tissue or cell solubilization was performed using cold radioimmunoprecipitation lysis buffer. The proteins were separated with $12 \%$ SDS-PAGE and transferred onto a polyvinylidene difluoride (PVDF) membrane, which was subsequently incubated with Tris buffered saline with Tween 20 containing $5 \%$ milk at room temperature for $3 \mathrm{~h}$. The PVDF membrane was then further incubated with rabbit polyclonal anti-Cdc42 (1:100; cat. no. ab64533), rabbit monoclonal anti-phospho-STAT3 (1:50; cat. no. ab76315), rabbit monoclonal anti-STAT3 (1:50; cat. no. ab32500) and rabbit monoclonal anti-GAPDH (1:50; cat. no. ab181602) primary antibodies (Abcam, Cambridge, $\mathrm{UK}$ ), respectively, at room temperature for $3 \mathrm{~h}$. Subsequent to being washed three times in phosphate-buffered saline (PBS) with Tween 20, the membrane was incubated with the goat anti-rabbit secondary antibody (Abcam) at room temperature for $40 \mathrm{~min}$. Chemiluminescent detection was carried out using an enhanced chemiluminescence kit (Pierce Chemical, Rockford, IL, USA), and the relative protein expression was analyzed using Image-Pro Plus software 6.0 (Media Cybernetics, Inc., Rockville, MD, USA). Relative protein expression was shown as the density ratio versus GAPDH.
Transfection. Transfection was performed using Lipofectamine 2000 (Invitrogen Life Technologies), according to the manufacturer's instructions. For the functional analysis of miR-195, bladder cancer T24 cells were transfected with scrambled miRNA as a negative control, miR-195 mimics or miR-195 inhibitor (Invitrogen Life Technologies). For the functional analysis of $\mathrm{Cdc} 42$, human bladder cancer T24 cells were transfected with Cdc42-specific small interfering RNA (siRNA) (Santa Cruz Biotechnology, Inc., Dallas, TX, USA) or pcDNA3.1-Cdc42 plasmid (Nlunbio, Changsha, China).

Dual-luciferase reporter assay. A mutant-type 3'-UTR of Cdc42 was produced using the QuikChange ${ }^{\mathrm{TM}}$ Site-Directed Mutagenesis kit (Stratagene, La Jolla, CA, USA). The wildand mutant-type 3'-UTRs of Cdc42 were inserted into the psiCHECK $^{\mathrm{TM}} 2$ vector (Promega Corp., Madison, WI, USA), respectively. Human bladder cancer T24 cells were cultured to $\sim 60 \%$ confluence in a 24 -well plate, and the T24 cells were then transfected with psiCHECK ${ }^{\mathrm{TM}} 2-\mathrm{Cdc}_{4}$ 2-3'-UTR or psiCHECK ${ }^{\mathrm{TM}}$ 2-mutant Cdc42-3'-UTR vector, respectively, with or without $100 \mathrm{~nm}$ miR-195 mimics, using Cellfectin ${ }^{\circledR}$ II Reagent (Invitrogen Life Technologies). After $48 \mathrm{~h}$ the dual-luciferase activities in each group were examined on an LD400 luminometer (Beckman Coulter, Fullerton, CA, USA). The activity of Renilla luciferase was normalized to that of firefly luciferase.

Cell proliferation assay. T24 cells in each group were seeded into a 96-well plate. Following culture for 48 h, MTT $(0.5 \mu \mathrm{g} / \mathrm{ml})$ was added into the medium for $1 \mathrm{~h}$ of incubation. The medium was then removed and the plate was washed three times in PBS, prior to the addition of $100 \mu \mathrm{l}$ dimethylsulfoxide to dissolve the precipitate. The absorbance at $570 \mathrm{~nm}$ was determined using a microplate reader (Bio-Rad, Hercules, CA, USA).

Statistical analysis. The results are expressed as the mean \pm standard deviation of three independent experiments. The statistical analysis of differences was performed using one-way analysis of variance with SPSS 17.0 software (SPSS, Inc., Chicago, IL, USA). $\mathrm{P}<0.05$ was considered to indicate a statistically significant difference.

\section{Results}

miR-195 expression is downregulated and Cdc42 expression is upregulated in bladder cancer tissues and cells. The expression level of miR-195 was determined in bladder cancer tissues, as well as their matched normal adjacent tissues, using RT-qPCR. As shown in Fig. 1A, miR-195 expression in the bladder cancer tissues was notably downregulated compared with that in the matched adjacent tissues. The expression of miR-195 was further examined in bladder cancer T24 cells, which showed that miR-195 expression was also downregulated in T24 cells compared with that in normal tissues.

The protein level of Cdc42 was determined in bladder cancer tissues and their matched adjacent normal tissues. Western blotting data showed that the protein level of Cdc42 was upregulated in bladder cancer tissues and T24 cells compared with that in normal tissues (Fig. 1B), suggesting that $\mathrm{Cdc} 42$ may play a suppressive role in bladder cancer. 
A

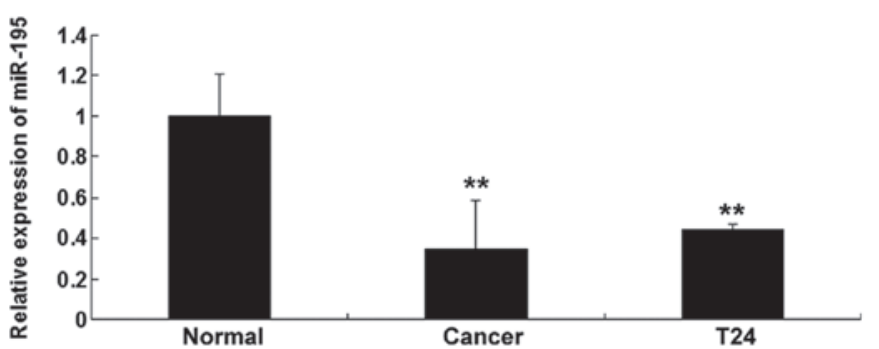

B
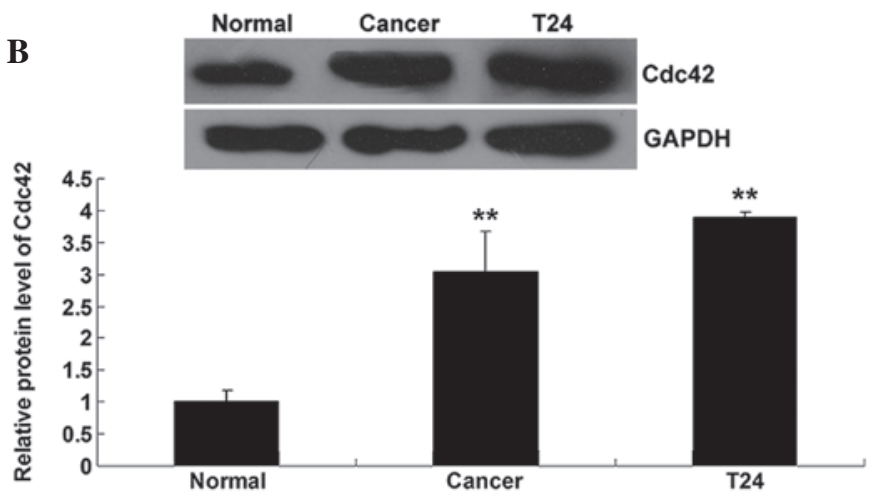

Figure 1. miR-195 expression is downregulated in bladder cancer tissues and T24 cells. (A) Reverse transcription-quantitative polymerase chain reaction analysis was performed to determine the expression of miR-195 in bladder cancer tissues (cancer) and their matched adjacent normal tissues (normal), as well as in bladder cancer T24 cells. ${ }^{* *} \mathrm{P}<0.01$ vs. normal. (B) Western blotting was performed to determine the protein level of Cdc42 in bladder cancer tissues (cancer) and their matched adjacent normal tissues (normal), as well as in bladder cancer T24 cells. ${ }^{* *} \mathrm{P}<0.01$ vs. normal. miR-195, microRNA-195; Cdc42, cell division control protein 42 homolog; GAPDH, glyceraldehyde 3-phosphate dehydrogenase.

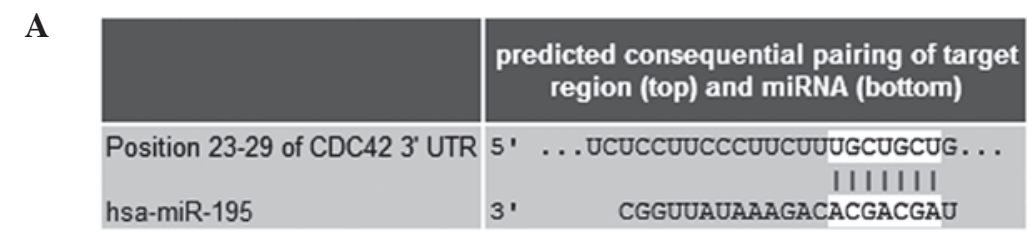

B

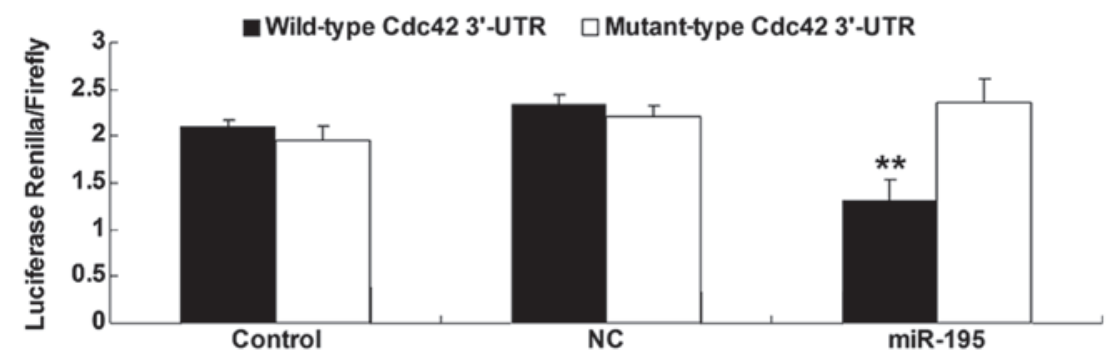

Figure 2. Identification of Cdc42 as a novel target of miR-195. (A) Bioinformatics analysis using TargetScan (http://www.targetscan.org/) revealed the putative seed region of miR-195 in the wild-type 3'-UTR of Cdc42. (B) Luciferase reporter assay was used to determine whether Cdc42 was a target of miR-195. Wild- and mutant-type 3'-UTRs of Cdc42 were subcloned into the psiCHECK ${ }^{\mathrm{TM}} 2$ reporter vector, respectively. PsiCHECK ${ }^{\mathrm{TM}} 2$-Cdc42-3'-UTR or psiCHECK $^{\text {TM }} 2$-mutant Cdc42-3'-UTR vector plus 50nM miR-195 or scramble negative control miRNA were co-transfected into T24 cells, respectively. " $\mathrm{P}<0.01$ vs. control. Control, cells without transfection of miRNA; NC, T24 cells transfected with scramble negative control miRNA; miR-195, microRNA-195; Cdc42, cell division control protein 42 homolog; UTR, untranslated region.

miR-195 directly binds to the 3'-UTR of Cdc42. Bioinformatics prediction was performed using TargetScan (http://www.targetscan.org/), which showed that the putative seed sequences for miR-195 at the 3'-UTR of Cdc42 were highly conserved (Fig. 2A). The wild and mutant types of Cdc42 3'-UTR were then generated, and dual-luciferase reporter assays were performed in bladder cancer T24 cells in order to verify whether Cdc42 is a direct target of miR-195. As shown in Fig. 2B, the Renilla/firefly value of luciferase activity was notably decreased only in bladder cancer T24 cells co-transfected with the wild-type 3'-UTR of Cdc42 and miR-195 mimics, indicating that Cdc42 is a direct target of miR-195 in bladder cancer T24 cells.

Cdc42 protein expression is negatively regulated by $\mathrm{miR}-195$ in bladder cancer T24 cells. To further determine the effects of miR-195 on Cdc42 expression in bladder cancer cells, bladder cancer T24 cells were transfected with miR-195 mimics, miR-195 inhibitor and scramble miRNA, respectively. As shown in Fig. 3, the protein level of Cdc42 was notably decreased in T24 cells transfected with miR-195 mimic, while increased in T24 cells transfected with miR-195 inhibitor, indicating that miR-195 negatively regulates the protein level of $\mathrm{Cdc} 42$ in bladder cancer T24 cells.

Cdc42/STAT3 signaling is involved in the miR-195-mediated inhibition of bladder cancer cell proliferation. As Cdc42/STAT3 signaling has been found to largely contribute to bladder cancer cell proliferation (14), and miR-195 can inhibit the protein expression of $\mathrm{Cdc} 42$ in bladder cancer T24 cells, we speculated that miR-195 could play a suppressive role in bladder cancer cell proliferation, via downregulation of Cdc42/STAT3 signaling. To verify this theory, bladder cancer T24 cells were transfected with miR-195 mimics or Cdc42-specific siRNA, or co-transfected with miR-195 mimics and $\mathrm{Cdc} 42$ plasmid, respectively. A cell proliferation 


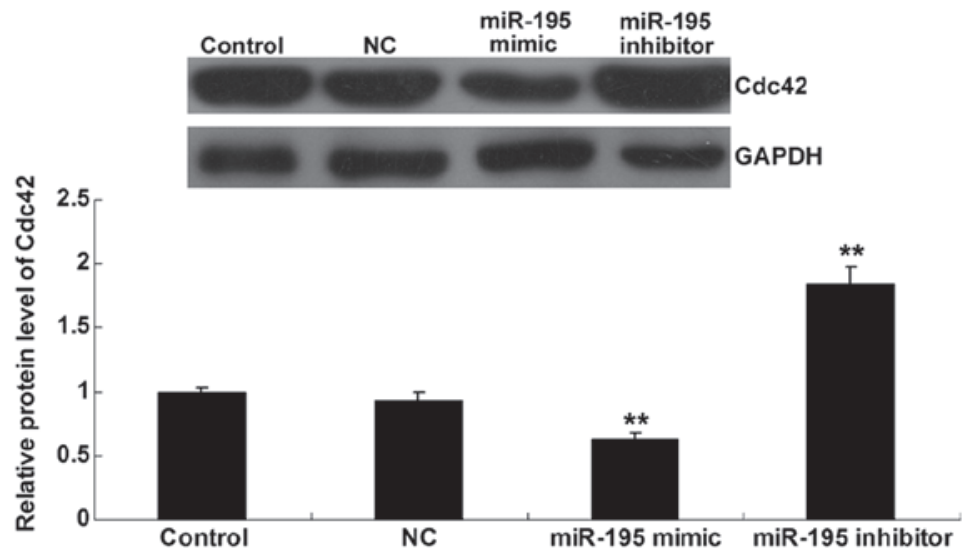

Figure 3. miR-195 negatively regulates the protein expression of Cdc42 in T24 cells. Western blot analysis was used to determine the protein level of Cdc 42 in T24 cells transfected with miR-195 mimic or miR-195 inhibitor, respectively. GAPDH was used as an endogenous reference. ${ }^{* *} \mathrm{P}<0.01$ vs. control. Control, T24 cells without any transfection; NC, T24 cells transfected with scramble miRNA; miR-195, microRNA-195; Cdc42, cell division control protein 42 homolog; GAPDH, glyceraldehyde 3-phosphate dehydrogenase.

$\mathbf{A}$

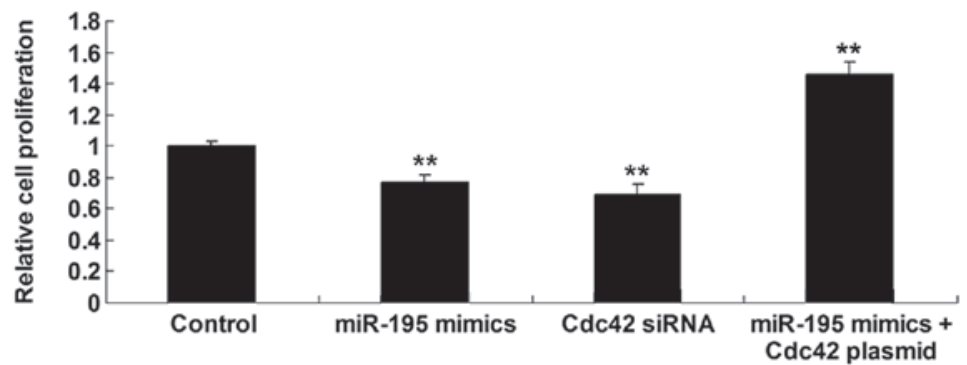

$\mathbf{B}$

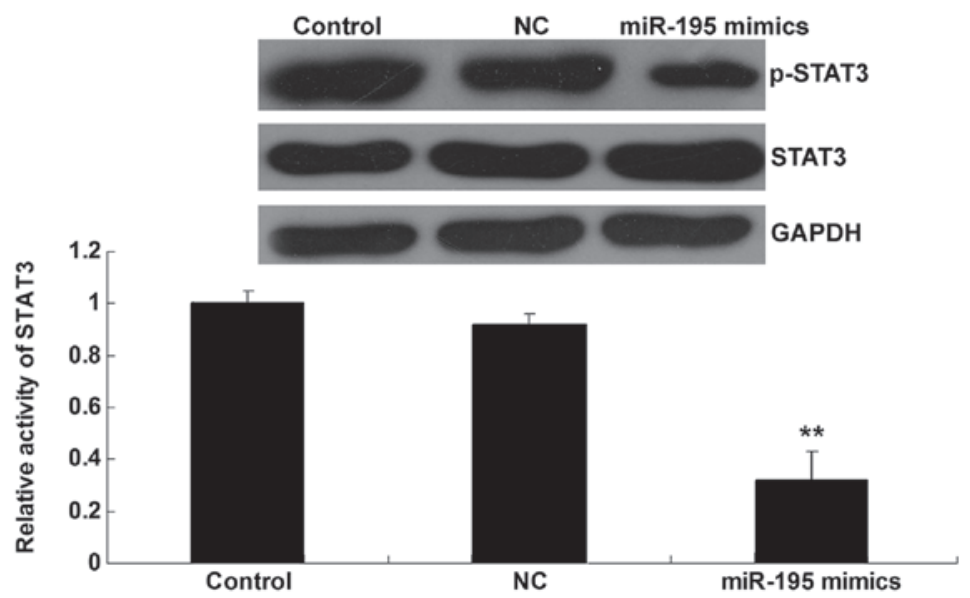

Figure 4. Cdc42/STAT3 signaling is involved in the miR-195-mediated inhibition of bladder cancer cell proliferation. (A) Cell proliferation assay was performed using T24 cells transfected with miR-195 mimics or Cdc42 siRNA, or co-transfected with miR-195 mimics and Cdc42 plasmid, respectively. ${ }^{* *} \mathrm{P}<0.01$ vs. control. (B) Western blotting assay was performed to determine the activation of STAT3 in T24 cells transfected with miR-195 mimics. ${ }^{* *} \mathrm{P}<0.01 \mathrm{vs}$. control. Control, T24 cells without any transfection; NC, T24 cells transfected with scramble miRNA; siRNA, small interfering RNA; p-STAT3, phospho-signal transducer and activator of transcription-3; miR-195, microRNA-195; Cdc42, cell division control protein 42 homolog; GAPDH, glyceraldehyde 3-phosphate dehydrogenase.

assay was then performed. It was found that upregulation of miR-195 and the inhibition of Cdc42 significantly inhibited T24 cell proliferation. In addition, the inhibitory effect of miR-195 upregulation on T24 cell proliferation was significantly reversed by the overexpression of Cdc42 (Fig. 4A). It was further confirmed that the activation of STAT3 was inhibited by miR-195 upregulation (Fig. 4B). Accordingly, we suggest that miR-195 inhibits bladder cancer cell proliferation at least partially by the inhibition of Cdc42/STAT3 signaling.

\section{Discussion}

In the present study, it was demonstrated that miR-195 was markedly downregulated, while the protein expression of 
Cdc42 was significantly increased, in bladder cancer tissues when compared with that in the matched normal adjacent tissues. Furthermore, Cdc42 was identified as a direct target of miR-195 in bladder cancer cells, and it was demonstrated that the protein level of $\mathrm{Cdc} 42$ was negatively regulated by miR-195. The present findings suggest that Cdc42/STAT3 signaling acts as a downstream effector in miR-195-mediated bladder cancer cell proliferation.

miRNAs have been demonstrated to act as mediators in various biological processes via modulation of the expression of target genes at a post-transcriptional level, and the dysregulation of miRNAs plays a key role in the development of various types of cancer $(15,16)$. In the present study, it was found that miR-195 expression was significantly downregulated in bladder cancer tissues compared with that in their matched normal adjacent tissues, which was consistent with other studies $(4,17)$. The first finding that miR-195 expression was notably downregulated in bladder cancer tissues when compared with that in normal bladder epithelium tissues was reported in a study by Ichimi et al (4). Han et al (17) then examined the expression profile of miRNAs using deep sequencing and qPCR, and showed that miR-195 expression was notably downregulated in bladder cancer tissues compared with that in matched histologically normal urothelium. More recently, Itesako et al (18) examined the miRNA expression signature of bladder cancer by deep sequencing, and revealed the functional significance of the miR-195/497 cluster. Based on these and the present findings, we suggest that miR-195 may be involved in the development and progression of bladder cancer.

A recent study revealed a detailed role of miR-195 in bladder cancer cells, and showed that miR-195 could suppress glucose uptake and the proliferation of bladder cancer T24 cells by inhibiting GLUT3 expression (10). In the present study, it was also found that miR-195 could inhibit bladder cancer T24 cell proliferation. Furthermore, Cdc42/STAT3 was revealed to be the downstream effector of miR-195 in bladder cancer T24 cells.

Cdc42 is a small GTPase of the Rho-subfamily. It has been well established that $\mathrm{Cdc} 42$ plays crucial roles in the regulation of various cellular functions, particularly cell cycle progression (19). More recently, the vital role of Cdc42 in the regulation of cancer cell proliferation, migration and invasion has been gradually revealed, and $\mathrm{Cdc} 42$ may become a promising target for the treatment of cancer $(13,20)$. In a study by Zins et al (21) it was demonstrated that a Ras-related C3 botulinum toxin substrate 1/Cdc42 GTPase-specific small molecule inhibitor could effectively suppress the growth of primary human prostate cancer xenografts and prolong survival in mice. Furthermore, it has been reported that $\mathrm{Cdc} 42$ expression is significantly upregulated in bladder cancer tissues compared with that in normal tissues, and $\mathrm{Cdc} 42$ silencing caused by siRNA can inhibit the phosphorylation of STAT3 and suppress the growth of bladder cancer cells, suggesting that $\mathrm{Cdc} 42$ may serve as a therapeutic target for the treatment of bladder cancer $(22,14)$. In the present study, it was demonstrated that miR-195 upregulation could inhibit $\mathrm{Cdc} 42$ protein expression in bladder cancer T24 cells. Further investigation showed that the activity of STAT3, a downstream effector of Cdc42, was also reduced following miR-195 upregulation, suggesting that miR-195 plays an inhibitory role in the regulation of Cdc42/STAT3 signaling.
STAT3 is a member of the STAT family of cytoplasmic transcription factors. Overactivation of STAT3 has been found in various types of cancer, suggesting that its overactivation may be closely associated with the development and progression of human malignancies (23). Evidence has revealed a close association and crosstalk between Cdc42 and STAT3 signaling, and upregulation of Cdc42 can further induce the activation of STAT3 (24). In addition, STAT3 has been shown to be required for the Cdc42-induced activation of nuclear factor- $\kappa \mathrm{B}$ signaling, which plays a key role in the regulation of cell survival and proliferation (25). In the present study, it was demonstrated that miR-195 upregulation inhibited bladder cancer T24 cell proliferation, accompanied by downregulation of Cdc42/STAT3 signaling, while restoration of Cdc42 reversed the effect of miR-195 upregulation and promoted the activity of STAT3. These findings suggest that Cdc42/STAT3 signaling acts as a downstream effector in the miR-195-mediated inhibition of bladder cancer cell proliferation.

In conclusion, the present study has identified Cdc42 as a direct target of miR-195 in bladder cancer cells, and suggests that miR-195 can inhibit bladder cancer cell proliferation at least partially via the inhibition of Cdc42/STAT3 signaling. Accordingly, miR-195 may be used as a promising therapeutic agent for bladder cancer.

\section{Acknowledgements}

This study was supported by the National Natural Science Foundation of China (no. H161981202005) and the State Scholarship Fund of China Scholarship Council (no. 201206370067).

\section{References}

1. Skeldon SC and Larry Goldenberg S: Bladder cancer: A portal into men's health. Urol Oncol 33: 40-44, 2014.

2. Ghafouri-Fard S, Nekoohesh L and Motevaseli E: Bladder cancer biomarkers: review and update. Asian Pac J Cancer Prev 15: 2395-2403, 2014.

3. Huang X, Liang M, Dittmar R and Wang L: Extracellular microRNAs in urologic malignancies: chances and challenges. Int J Mol Sci 14: 14785-14799, 2013.

4. Ichimi T, Enokida H, Okuno Y, et al: Identification of novel microRNA targets based on microRNA signatures in bladder cancer. Int J Cancer 125: 345-352, 2009.

5. Yoshino H, Seki N, Itesako T, Chiyomaru T, Nakagawa M and Enokida H: Aberrant expression of microRNAs in bladder cancer. Nat Rev Urol 10: 396-404, 2013.

6. Chabre O, Libé R, Assie G, et al: Serum miR-483-5p and miR-195 are predictive of recurrence risk in adrenocortical cancer patients. Endocr Relat Cancer 20: 579-594, 2013.

7. Yongchun Z, Linwei T, Xicai W, et al: MicroRNA-195 inhibits non-small cell lung cancer cell proliferation, migration and invasion by targeting MYB. Cancer Lett 347: 65-74, 2014

8. Tahiri A, Leivonen SK, Lüders T, et al: Deregulation of cancer-related miRNAs is a common event in both benign and malignant human breast tumors. Carcinogenesis 35: 76-85, 2014.

9. Yang G, Wu D, Zhu J, et al: Upregulation of miR-195 increases the sensitivity of breast cancer cells to Adriamycin treatment through inhibition of Raf-1. Oncol Rep 30: 877-889, 2013.

10. Fei X, Qi M, Wu B, Song Y, Wang Y and Li T: MicroRNA-195-5p suppresses glucose uptake and proliferation of human bladder cancer T2 4 cells by regulating GLUT3 expression. FEBS Lett 586: 392-397, 2012.

11. Lin Y, Wu J, Chen H, et al: Cyclin-dependent kinase 4 is a novel target in micoRNA-195-mediated cell cycle arrest in bladder cancer cells. FEBS Lett 586: 442-447, 2012. 
12. Dogini DB, Pascoal VD, Avansini SH, Vieira AS, Pereira TC and Lopes-Cendes I: The new world of RNAs. Genet Mol Biol 37 (1 Suppl): 285-293, 2014

13. Stengel $\mathrm{K}$ and Zheng Y: Cdc42 in oncogenic transformation, invasion, and tumorigenesis. Cell Signal 23: 1415-1423, 2011.

14. Wu F, Chen Y, Li Y, Ju J, Wang Z and Yan D: RNA-interference-mediated Cdc42 silencing down-regulates phosphorylation of STAT3 and suppresses growth in human bladder-cancer cells. Biotechnol Appl Biochem 49: 121-128, 2008.

15. Ambros V: The functions of animal microRNAs. Nature 431: 350-355, 2004

16. Baer C, Claus R and Plass C: Genome-wide epigenetic regulation of miRNAs in cancer. Cancer Res 73: 473-477, 2013.

17. Han Y, Chen J, Zhao X, et al: MicroRNA expression signatures of bladder cancer revealed by deep sequencing. PLoS One 6: e18286, 2011

18. Itesako T, Seki N, Yoshino H, et al: The microRNA expression signature of bladder cancer by deep sequencing: The functional significance of the miR-195/497 cluster. PLoS One 9: e84311, 2014

19. Ni S, Hu J, Duan Y, Shi S, Li R, Wu H, Qu Y and Li Y: Down expression of LRP1B promotes cell migration via RhoA/Cdc42 pathway and actin cytoskeleton remodeling in renal cell cancer. Cancer Sci 104: 817-825, 2013.
20. Ma L, Deng X, Wu M,Zhang G and Huang J: Down-regulation of miRNA-204 by LMP-1 enhances CDC42 activity and facilitates invasion of EBV-associated nasopharyngeal carcinoma cells. FEBS Lett 588: 1562-1570, 2014.

21. Zins K, Lucas T, Reichl P, Abraham D and Aharinejad S A Rac1/Cdc42 GTPase-specific small molecule inhibitor suppresses growth of primary human prostate cancer xenografts and prolongs survival in mice. PLoS One 8: e74924, 2013.

22. Volanis D, Zaravinos A, Kadiyska T, Delakas D, Zoumpourlis V and Spandidos DA: Expression profile of Rho kinases in urinary bladder cancer. J BUON 16: 511-521, 2011.

23. Raptis L, Arulanandam R, Geletu M and Turkson J: The R(h)oads to Stat3: Stat3 activation by the Rho GTPases. Exp Cell Res 317: 1787-1795, 2011.

24. Arulanandam R, Vultur A, Cao J, et al: Cadherin-cadherin engagement promotes cell survival via Rac1/Cdc42 and signal transducer and activator of transcription-3. Mol Cancer Res 7: 1310-1327, 2009

25. Debidda M, Wang L, Zang H, Poli V and Zheng Y: A role of STAT3 in Rho GTPase-regulated cell migration and proliferation. J Biol Chem 280: 17275-17285, 2005. 